submitted to PRL

\title{
Enhanced Proton Acceleration by an Ultrashort Laser Interaction with Structured Dynamic Plasma Targets
}

\author{
A. Zigler, ${ }^{1}$ S. Eisenman, ${ }^{1}$ M. Botton, ${ }^{1,}{ }^{*}$ E.Nahum, ${ }^{1}$ E. Schleifer, ${ }^{1}$ A. Baspaly, ${ }^{1}$ \\ Y.Pomerantz, ${ }^{1}$ F. Abicht, ${ }^{2}$ J. Branzel, ${ }^{2}$ G.Priebe, ${ }^{2}$ S.Steinke, ${ }^{2}$ A. Andreev, ${ }^{2}$ M. \\ Schnuerer, ${ }^{2}$ W.Sandner, ${ }^{2}$ D. Gordon, ${ }^{3}$ P. Sprangle, ${ }^{3}$ and K.W.D.Ledingham ${ }^{4}$ \\ ${ }^{1}$ Racah Institute of Physics, The Hebrew University of Jerusalem Jerusalem, ISRAEL \\ ${ }^{2}$ Max Born Institute, Berlin ,Germany \\ ${ }^{3}$ Plasma Physics Division, Naval Research Lab, Washington, D.C. 20375, USA \\ ${ }^{4}$ University of Strathclyde, Glasgow G4 ONG, Scotland, UK
}

(Dated: November 11, 2018)

\begin{abstract}
We experimentally demonstrate a notably enhanced acceleration of protons to high energy by relatively modest ultrashort laser pulses and structured dynamical plasma targets. Realized by special deposition of snow targets on sapphire substrates and using carefully planned pre-pulses, high proton yield emitted in a narrow solid angle with energy above $21 \mathrm{MeV}$ were detected from a $5 \mathrm{TW}$ laser. Our simulations predict that using the proposed scheme protons can be accelerated to energies above $150 \mathrm{MeV}$ by $100 \mathrm{TW}$ laser systems.
\end{abstract}

PACS numbers: 52.38.Kd, 41.75.Jv, 52.35.Mw, 52.65.Rr

Proton acceleration by the interaction of an ultra high intensity laser beam with matter has several wide prospective applications including cancer treatment, astrophysics in the lab, nuclear physics, and material sciences (see $[1,2]$ for review). Over the years several promising acceleration schemes were proposed and demonstrated, such as Target Normal Sheath Acceleration (TNSA) [3-5], Radiation Pressure Acceleration (RPA) [6,7], Break Out Afterburner (BOA) [8], and collisionless shock acceleration [9]. Variations of these schemes including mass-limited targets [10] nanostructure targets [11-13] aimed at increasing the efficiency of the interaction were also considered. Some of these schemes require exceedingly a high intensity of the laser beam in order to initiate the process, and most of them require a laser energy exceeding 1PW level (on target) in order to accelerate the protons to energies about $150 \mathrm{MeV}$ required by medical applications. Furthermore, all these schemes pose the same requirement of the laser system to have as low as possible energy stored in the prepulse. The main reason is that these accelerating schemes are optimized for an interaction between the main pulse and a cold solid-density target, and are strongly degraded as the main laser pulse interacts with a pre-heated (or even ionized) target. It was recently shown that preformed plasma may be beneficial to the acceleration process $[14,15]$. Nevertheless, the majority of the experiments still aim at cold targets with as low as possible pre-pulse. Accordingly, achieving such a low energy content in the pre-pulse requires a contrast ratio of the order of $10^{-11}$ for a $100 \mathrm{TW}$ laser and even higher for more energetic systems, which is a real experimental challenge.

In this letter we report for the first time on acceleration of proton bunches to energies above $21 \mathrm{MeV}$ by a

\footnotetext{
* Corresponding author: bdmoti@phys.huji.ac.il
}

5TW ultrashort (50fs) laser pulse. We introduce an alternative approach to the laser-based proton acceleration quest by using a moderate power $(<10 \mathrm{TW})$ laser system, and carefully produced microstructured snow targets [16-17]. Our experimental results show that the energy of the accelerated protons scales with the power of the laser according to the $E_{p} \propto P_{L}^{1 / 2}$ rule which is obtained here for significantly lower laser powers than the traditional schemes. Numerical 2D PIC code simulations of the interaction process reproduce the experimentally obtained scaling law and predict the possibility of accelerating protons to $150 \mathrm{MeV}$ with a laser power of about $100 \mathrm{TW}$. This notably increased proton energy is attributed to a combination of three mechanisms. First is the localised enhancement of the laser field intensity near the tip of the microstructured whisker. This causes an increased electronic charge repulsion out of the whisker. Second is a mass-limited like phenomena, namely the absence of high density cold electron cloud in the vicinity of the whisker which can compensate for the expelled electrons. The heated electrons remain in the vicinity of the positively charged whisker, producing strong accelerating electrostatic fields and pulling the protons out. Third is the Coulomb explosion of the positively charged whisker, adding a longtime acceleration to the protons. As our innovative microstructured snow scheme requires the interaction of the laser with a structured dynamical plasma target, it also relaxes the requirements of high contrast ratio of the laser system, and facilitates the production of the target.

Two separate laser systems were used to obtain the results reported here. One is the laser at the High-Intensity Laser Lab at the Hebrew University of Jerusalem (HUJI) and the other is the laser system at the Max Born Institute at Berlin (MBI). The HUJI system is a $2 \mathrm{TW}, 50 \mathrm{fs}$ pulse length laser operating at a central wavelength of $0.8 \mu$. The spot size (FWHM) as small as $20 \mu m^{2}$. The 


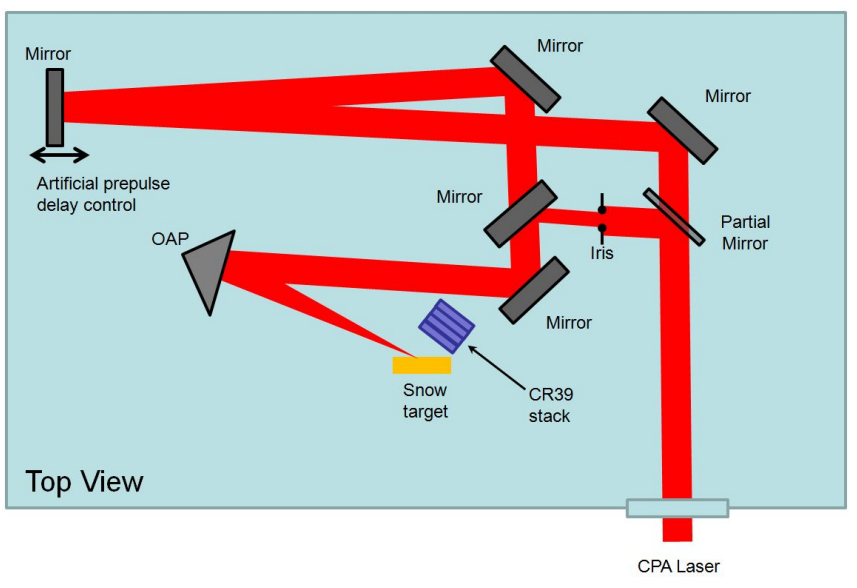

FIG. 1. The experimental setup

contrast ratio of the HUJI system is about 1000 dictated by the leakage of the regenerative amplifier, thus the prepulse is shaped similarly to the main pulse and arrives on the target approximately 10nsec before the main pulse. The MBI system is a $30 \mathrm{TW}, 70 \mathrm{fs} 0.8 \mu$ laser. The beam is focused to a spot size of $5 \mu$ on target. The contrast ratio of the MBI system is about $10^{-8}$, and the pre-pulse is a pedestal like shape. In order to obtain the same pre-plasma conditions in both institutes, the main beam at the MBI system is split and a pre-pulse is artificially formed. The experiments at the MBI site were carried out using a $0.03 \mathrm{~mJ}$ prepulse $6 \mathrm{~ns}$ ahead of the main pulse.

A schematic diagram of the experimental setup in both institutes is shown in Fig. 1. The laser beam strikes the target at an angle $60^{\circ}$ to the normal. The target is a liquid nitrogen cooled sapphire substrate on which water vapor is deposited. The size and density of the snow pillars as well as the surface roughness are determined by various parameters such as pressure, flow and temperature.

Fig. 2 shows a typical snow targets as imaged by a scanning electron microscope. The snow surface can be characterised as a highly structured surface with essentially three roughness scales: a) pillars of about $100 \mu$, b) spikes of about $10 \mu$ on top of them and c) whiskers of about $1 \mu$ on the spikes. The distribution of the pillars and spikes is controlled by seeding and fixing the flow of the vapor. The red circle in Fig 2 schematically represents the laser spot (pre-pulse and main pulse) for a typical shot. Based on our measurements of the laser spot characteristics, we estimate that the laser spot interacts with few (typically less than 5) whiskers.

The pre pulse meets the whiskers and vaporizes part of them. The time delay between the pre and main pulse is about 10ns, during which the plasma can freely expand and a highly non-uniform plasma cloud is formed. By the time of its arrival, the main pulse meets this highly structured dynamic plasma cloud and interacts with it to produce the accelerated protons. The main diagnostics consists of stacks of CR39 film, each $25 \mathrm{~mm}$ diam-
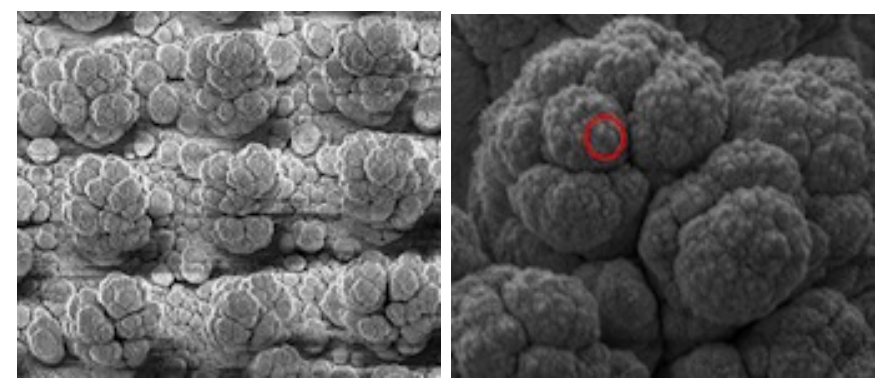

FIG. 2. Scanning electron microscope images of the snow targets. Red circle represents the laser spot.
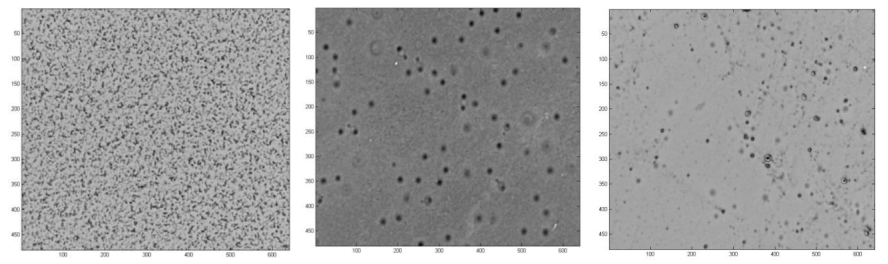

FIG. 3. Processed CR39 showing marks of protons with energy $>1 \mathrm{MeV}$. (left), energy $>13 \mathrm{MeV}$. (middle) energy $>20 \mathrm{MeV}$. (right)

eter. The thickness of the CR39 is $1.5 \mathrm{~mm}$. In front of the first CR39 film we positioned a $13 \mu$ Aluminum foil which prevents the plasma plume emitted in the process to damage the films. The detector films are positioned at a distance of about $2.5 \mathrm{~cm}$ from the target at an angle of 450. The setup enables sufficient energy and spatial resolution. The CR39 plates are replaced every several laser shots (typically 3-5) and the exposed films are etched using the conventional methods. Analysis of the processed CR39 consisted on automated counting of the pits and comparing the resulted number to unexposed areas on the same film and on a reference (processed) film. Exposed areas in all the films showed clear signal far above the noise level, which is unquestionably the result of accelerated protons. Fig. 3 shows a $100 \mu$ x $100 \mu$ area of three processed CR39 films. The first plate was uniformly covered by proton tracks. Considering the thickness of the foil that covered the stack it is concluded that the tracks are produced by protons of energy above $1 \mathrm{MeV}$. The second CR39 detector was covered by a lower density but still uniform distribution of tracks. These are attributed to protons of energy above $13 \mathrm{MeV}$. The tracks marked on the third plate are the result of protons with energy above $20 \mathrm{MeV}$. Here the density is not uniform and bunches can be seen.

We have repeated the experiment with various laser power levels to produce the energy scaling of the most energetic protons accelerated of the snow targets (Fig. 4). The fitted scaling from this data set of this novel acceleration scheme is $E_{p} \propto P_{L}{ }^{0.58}$, and is obtained with lower laser power levels. Notice that unlike the traditional TNSA scheme there is no clear distinction of back and front surface as the laser does not cross the target but 


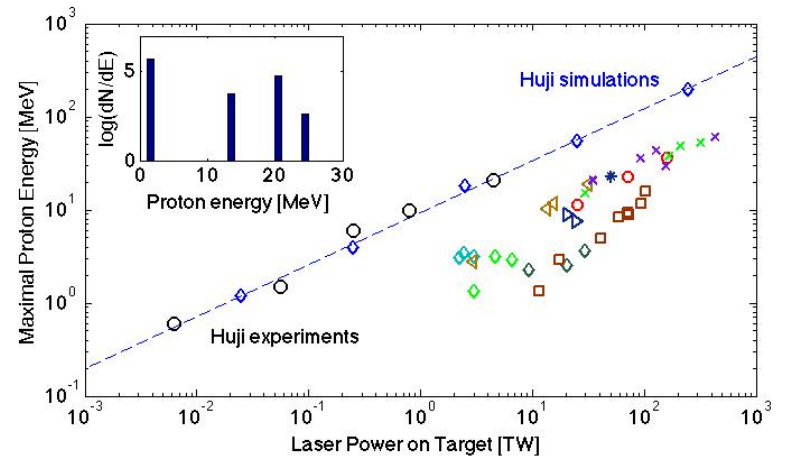

FIG. 4. Energy scaling of the accelerated protons as a function of the laser power on target. Various points describe data obtained by many contributors (See ref [18] for details)

interacts with its micro structured surface and the plasma cloud generated there by the pre pulse. At this point we note that when the pre-pulse was not present and the main pulse interacted directly with the snow surface, the resulted protons were accelerated to a much lower energy level, down to the traditional scaling of the TNSA scheme. A count of the total number of tracks on the CR39 detector plates is shown in the inset of Figure 4. The measured energy spectrum is not a monotonically decreasing function of the energy (within the available resolution) and a clear peak is evident at energies around $20 \mathrm{MeV}$. We also found that the most energetic protons were found on the CR39 detectors at a location which is close to the position of a reflected laser field for a planar target.

The TURBOWAVE particle in cell (PIC) [19] simulations of the unique acceleration scheme presented here are based on the one dimensional model presented in our previous work [17] which demonstrated the significant enhancement by the local plasma density near the whisker tip. Considering the size scales of the target and the laser spot size we focus our study on the interaction of the laser with a single snow whisker. Following the pre-pulse illumination, the whisker is partially vaporized and ionized hence a non uniform plasma cloud (protons, triplyionized oxygen and electrons) is formed.We model this highly structured dynamic plasma as an ellipsoid. We estimate that the high $\left(\sim 100 n_{c r}\right.$, where $n_{c r}=1.1 \cdot 10^{21} / \lambda^{2}$ is the traditional critical density) density portion of the whisker is an ellipsoid of the order of $0.1-0.2 \mu$ width (minor axis) and $1-2 \mu \mathrm{l}$ ength (major axis). Estimates of the density gradients of the plasma cloud (supported by a $1 \mathrm{D}$ hydrocode Hyades [20]) setthe critical density contour to be an ellipsoid of the order of $1-2 \mu$ width (minor axis) and $10 \mu$ length (major axis). The linearly polarized laser has a spot size of $5 \mu$ and is taken to hit the plasma cloud at an angle of $45^{\circ}$ with respect to the major axis. The laser pulse is $88 \mathrm{fs}$ total duration with a linear rise time and fall time of $32 \mathrm{fs}$ each. Further simplification is achieved by eliminating one dimension and setting the whisker to be independent of the y coordinate
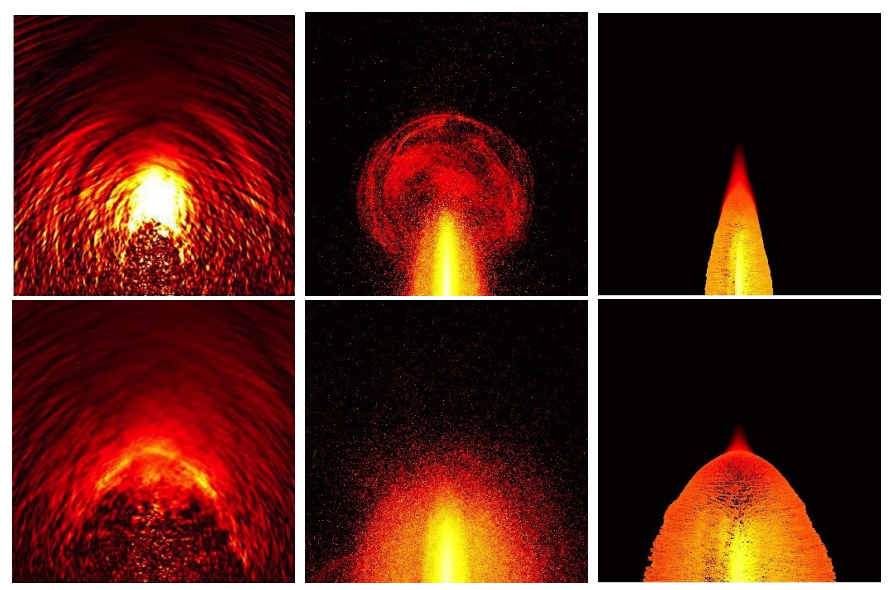

FIG. 5. Spatial distribution of protons (right), electron (middle), and electric field intensity along the major axis (left) at $220 \mathrm{fs}$ (top row) and $440 \mathrm{fs}$ (bottom row). All frames are $40 \mu \times 40 \mu$.Colormap scale for density are logarithmic normalized to critical density, and for field intensity are in normalized units $\left(a_{0}=e E / m c \omega\right)$

(hence it becomes a wedge), keeping the polarization of the laser in the $\mathrm{x}-\mathrm{z}$ plane. The $2 \mathrm{D}$ model is expected to reproduce the energy scaling of the accelerated protons but not the spatial distribution which will be strongly dependent on the angle between the polarized field and the major axis of the plasma ellipsoid as well as other neighboring whisker which affect the propagation of the laser field. The cell size is $0.05 \mu \times 0.05 \mu$, and the simulation region is $200 \mu \times 200 \mu$.

The interaction and acceleration process is roughly separated into three phases. The first phase, which is approximately the laser pulse duration, the laser drives the electrons out of the plasma ellipsoid. These electrons are accelerated by the laser's ponderomotive potential and part of them escape out of the plasma cloud leaving the heavy highly charged oxygen atoms and the protons behind. The curved equal density surfaces affect the ponderomotive potential force. Unlike the TNSA target, there is no clear distinction between back and front surfaces as they are mixed over the whisker tip. The second phase is a short time after the passage of the laser pulse, approximately 2-3 pulse duration. The top row of Fig. 5 shows the density of the protons (right) electrons (middle) and and the electric field along the major axis (left) at 220fs. Here we see the electron accumulation near the tip of the whisker. The protons starts to react to the accelerating field. The accumulation of the electrons by the tip produces a strong electrostatic field. The third and last phase is long time acceleration, around 36 laser pulse duration. The electron accumulation near the whisker tip is reduced but charge separation between electrons and ions is maintained. Fig 5 bottom row shows the density of proton, electron, and electric field along the major axis at $440 \mathrm{fs}$. The charge separation between the hot electrons and the protons generates an accelerating 
field while the un-neutralized heavier oxygen ions remain behind and add a pushing field. The highest energy of the protons for this simulation was found to be $15 \mathrm{MeV}$. The scaling law of figure 4 was obtained by repeating the simulations with changed laser power.

In conclusion, we have experimentally demonstrated acceleration of protons to energy up to $25 \mathrm{MeV}$ by a modest power $(\sim 5 T W)$ laser. The acceleration scheme is based on a highly structured dynamic plasma target which is produced by a pre-pulse illumination of a snow target. The energy scaling resembles the power law of planar targets but obtained with a much lower laser energy. The proton yield is of the order of $10^{6}$ protons per shot with an angular distribution of the high energy component of protons is $(\sim 0.1$ radians $)$. PIC simulations reproduce the energy scaling and predict that by using $100 \mathrm{TW}$ laser, protons can be accelerated to $150 \mathrm{MeV}$ level. This is a considerable improvement over alternative acceleration schemes which requires a higher energy laser system with an extremely high contrast ratio.
[1] K. W. D. Ledingham, W. Galster, New Journal of Phys., 12, 045005, (2010).

[2] H. Daido, M. Nishuichi, A. S. Pirozhkov, Rep. Prog. Phys., 75, 056401, (2012).

[3] S. Hatchet et al., Phys. Plasma, 7, 2076, (2000).

[4] S. C. Wilks et al., Phys. Plasma, 8, 542, (2001).

[5] S. A. Gaillard et al., in Proceedings of the APS 51st Annual Meeting of the Division of Plasma Physics, (APS, NewYork, 2009), Abstract No. G06.003.

[6] T. Esirkepov et al., Phys. Rev. Lett., 92, 175003, (2004).

[7] B. M. Hegelich et al., Nature (London), 439, 441, (2006).

[8] A. Henig et al., Phys. Rev. Lett., 103, 045002, (2009).

[9] D. Haberberger et al., Nature Phys., 8, 95, (2011).

[10] S. Steinke et al., Laser Part. Beams, 28, 215,(2010).

[11] H. Schwoerer et al., Nature (London), 439, 445, (2006).
[12] D. Margarone et al., Phys. Rev. Lett., 109, 234801,(2012).

[13] A. A. Andreev et al., Phys. Plasma, 18, 103103, (2011).

[14] A. R. Holdunkar, N. K. Gupta, Phys. Plasma, 15, 123104, (2008).

[15] T. Plachan et al., Appl. Phys. Lett., 90, 041501, (2007).

[16] T. Palchan et al., Appl. Phys. Lett., 91, 251501, (2007).

[17] A. Zigler et al., Phys. Rev. Lett., 106, 134801, (2011).

[18] K.Zeil et al., New Journal of Physics, 12,045015, (2010).

[19] D. F. Gordon, W. Mori, T. M. Antonsen Jr., IEEE Trans. Plasma Sci., 28, 1135, (2000); D. F. Gordon, IEEE Trans. Plasma Sci., 35, 1486, (2007).

[20] J. T. Larson, in W. Goldstein, C. Hooper, J. Gauthier, J. Seely, R. Lee Eds.., Radiative Properties of Hot Dense Matter, World Scientific, Singapore, 1991. 